\title{
Further Evidence of a Recessive Variant in COL1A1 as an Underlying Cause of Ehlers-Danlos Syndrome: A Report of a Saudi Founder Mutation
}

\author{
Ahmad Almatrafi ${ }^{1}$ Jamil A. Hashmi ${ }^{2}$ Fatima Fadhli ${ }^{3} \quad$ Asma Alharbi $^{2}$ Sibtain Afzal ${ }^{4}$ \\ Khushnooda Ramzan ${ }^{5}$ Sulman Basit ${ }^{2}$
}

${ }^{1}$ Department of Biology, College of Science, Taibah University, Almadinah Almunawwarah, Saudi Arabia

${ }^{2}$ Center for Genetics and Inherited Diseases, Taibah University Almadinah Almunawwarah, Medina, Kingdom of Saudi Arabia

${ }^{3}$ Department of Genetics, Madinah Maternity and Children Hospital, Medina, Kingdom of Saudi Arabia

${ }^{4}$ Faculty of Allied and Health Sciences, Imperial College of Business Studies, Lahore, Pakistan

${ }^{5}$ Department of Genetics, King Faisal Specialist Hospital and Research Centre, Riyadh, Kingdom of Saudi Arabia

\begin{abstract}
Address for correspondence Sulman Basit, MPhil, PhD, Center for Genetics and Inherited Diseases, Taibah University Almadinah Almunawwarah, Medina 42318, Kingdom of Saudi Arabia (e-mail: sbasit.phd@gmail.com).
\end{abstract}

\begin{abstract}
Keywords

- COL1A1 homozygous mutation

- Ehlers-Danlos syndrome

- founder mutation

- Saudi family

Ehlers-Danlos syndrome (EDS) is a group of clinically and genetically heterogeneous disorder of soft connective tissues. The hallmark clinical features of the EDS are hyperextensible skin, hypermobile joints, and fragile vessels. It exhibits associated symptoms including contractures of muscles, kyphoscoliosis, spondylodysplasia, dermatosparaxis, periodontitis, and arthrochalasia. The aim of this study is to determine the exact subtype of EDS by molecular genetic testing in a family segregating EDS in an autosomal recessive manner. Herein, we describe a family with two individuals afflicted with EDS. Whole exome sequencing identified a homozygous missense mutation (c.2050G > A; p.Glu684Lys) in the COL1A1 gene in both affected individuals, although heterozygous variants in the COL1A1 are known to cause EDS. Recently, only one report showed homozygous variant as an underlying cause of the EDS in two Saudi families. This is the second report of a homozygous variant in the COL1A1 gene in a family of Saudi origin. Heterozygous carriers of COL1A1 variant are asymptomatic. Interestingly, the homozygous variant identified previously and the one identified in this study are same (c.2050G >A). The identification of a unique homozygous mutation (c.2050G > A) in three Saudi families argues in favor of a founder effect.
\end{abstract}

\section{Introduction}

Ehlers-Danlos syndrome (EDS) is an inherited disorder of connective tissues and comprises a wide range of clinical conditions involving skin, joints, and vessels. EDS manifests as joint hypermobility, skin hyperextensibility, and vascular fragility. ${ }^{1,2}$ EDS is phenotypically heterogeneous and a variety of other associated clinical features have been reported, includ-

published online

February 1, 2021
DOI https://doi.org/

10.1055/s-0041-1722873.

ISSN 2699-9404. ing severe periodontitis, multiple congenital contractures, ocular malformations, kyphoscoliosis, and arterial and intestinal ruptures. ${ }^{3-7}$

Inheritance pattern in EDS is both an autosomal recessive as well as an autosomal dominant. The disorder is genetically heterogeneous and at least six autosomal dominant and seven autosomal recessive forms of the EDS have been identified and, except in one case, the corresponding genes have been

\section{(c) 2021. The Author(s).}

This is an open access article published by Thieme under the terms of the Creative Commons Attribution License, permitting unrestricted use, distribution, and reproduction so long as the original work is properly cited. (https://creativecommons.org/licenses/by/4.0/)

Georg Thieme Verlag KG, Rüdigerstraße 14, 70469 Stuttgart, Germany 
discovered. The autosomal dominant form of the EDS have been shown to result from mutations in the genes COL1A1, COL1A2, COL3A1, COL5A1, COL5A2, COL12A1,C1R, and C1S..$^{5,8-14}$ Whereas mutations in B4GALT7, B3GALT6, SLC39A13, ADAMTS2, TNXB, PLOD1, FKBP14 ZNF469, PRDM5 CHST14, DSE, and AEBP1 genes have been shown to cause autosomal recessive form of the EDS. ${ }^{15-24}$ Recently, Alazami et al have shown that homozygous mutation in the COL1A1 gene can also lead to an autosomal recessive form of EDS. ${ }^{23}$ Although, mutations in 19 different genes have been identified as an underlying cause of the 12 different EDS types, much remains to be determined clinically and molecularly about EDS phenotype and EDS like spectrum. ${ }^{25}$

Here, we presented a five generations consanguineous Saudi family with suspected symptoms of EDS. Considering the huge genetic heterogeneity, whole exome sequencing strategy was undertaken. Genetic data analysis identified a homozygous mutation in the COL1A1 as an underlying cause of EDS in the family.

\section{Materials and Methods}

\section{Ethical Approval and Sample Collection/Pedigree Information}

Ethical approval to commence the work was obtained from the ethical review committee of Taibah University Almadinah Almunawarah, Kingdom of Saudi Arabia. A 5-generation Saudi consanguineous family with two affected individuals was referred to the Center for Genetics and Inherited Diseases (CGID) from Madinah Maternity and Children Hospital $(\mathrm{MMCH})$ by a pediatric consultant for detailed molecular investigation. Informed written consents were taken from all the members of the family; parents gave consents in case of children. Family history of the disease was enquired from the parents, and a pedigree was drawn based upon the information taken from elders of the family ( $\mathbf{- F i g . 1 A}$ ).

\section{Clinical Evaluation}

A complete clinical checkup of the affected members was conducted at $\mathrm{MMCH}$ by a pediatric consultant. Skin and musculoskeletal system was evaluated. A Beighton score was used to assess the joint hypermobility. Full blood count and echocardiography were performed.

\section{DNA Extraction}

Approximately $5 \mathrm{~mL}$ peripheral blood sample was collected from affected as well as unaffected members of the family in EDTA blood vacutainers. Genomic DNA from these blood samples were extracted using QIAquick DNA extraction kit. Nanodrop spectrophotometer and gel electrophoresis were used to evaluate the quality and integrity of the extracted DNA, respectively.

\section{Whole Genome Single Nucleotide Polymorphism (SNP) Genotyping}

Whole genome SNP genotyping array was performed using Illumina iScan platform and HumanOmni $2.5 \mathrm{M}$ bead chip, genotyping 2.5 million SNPs. Approximately 200 ng genomic DNA of two affected and two unaffected members were taken for genotyping as per protocol described elsewhere. ${ }^{26,27}$ Illumina genome studio and homozygosity mapper ${ }^{28,29}$ were employed to detect common regions sharing homozygosity amongst the affected members.

\section{Whole Exome Sequencing (WES)}

In an attempt to identify the underlying genetic variant causing EDS, entire coding region (whole exome) was sequenced using Nextera rapid capture exome kit and Illumina NextSeq 500 machine. A total of $70 \mathrm{ng}$ of the genomic DNA from both the affected members were used as a starting material, while library preparation and exome enrichment were done using Nextera rapid capture exome kit. Detailed protocol used for WES was the same as described elsewhere. ${ }^{30}$

\section{Candidate Gene Validation by Sanger Sequencing}

Genetic analyzer ABI3500 was used to validate the genetic variant discovered by WES. Primer $3^{31}$ software was used to design the primers from the flanking regions of the candidate variants. Detailed protocol that was used for Sanger validation
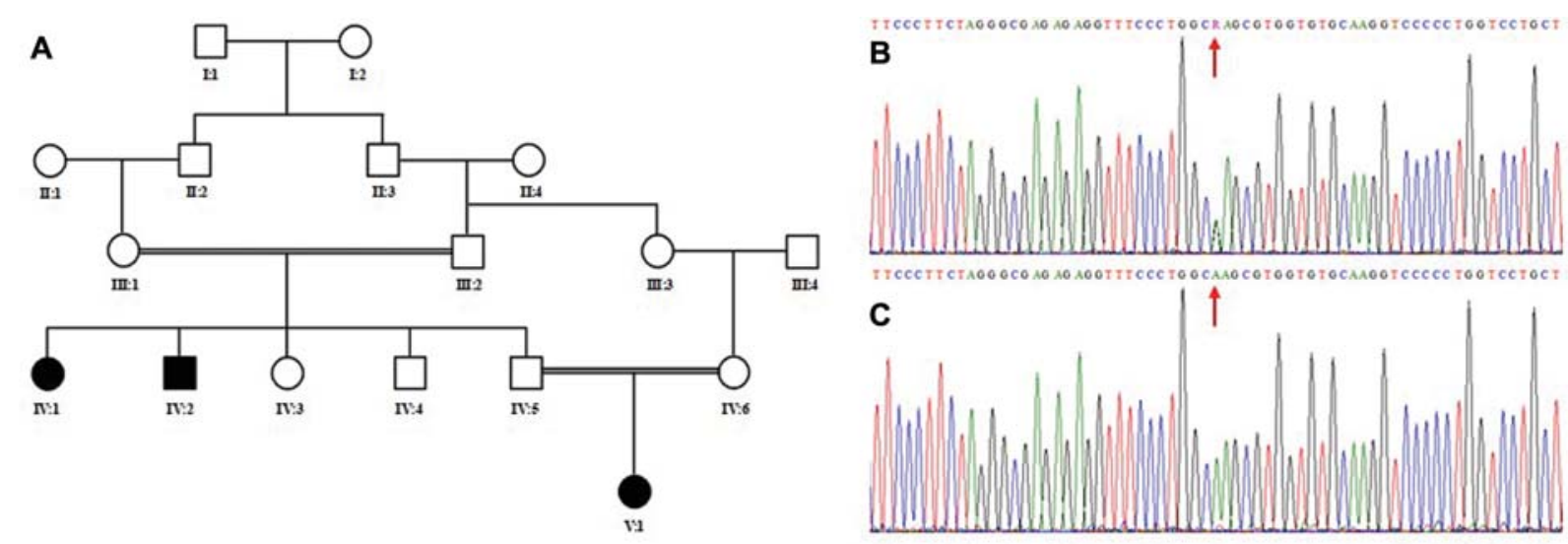

Fig. 1 (A) Pedigree of the family investigated in the present study showing autosomal recessive inheritance pattern. Double lines indicate consanguineous marriages. Filled symbols represent affected individuals. (B, C) Partial sequence of the exon 31 of the COL1A1 gene obtained by Sanger sequencing. Upper panel (B) shows chromatogram of the carrier and lower panel (C) shows chromatogram of the affected individuals of the family. Red arrow indicates the position of the mutation. 
was the same as described elsewhere. ${ }^{32,33}$ The sequenced variants were identified using BIOEDIT sequence alignment editor version 6.0.7 (Ibis bioSciences Inc.; CA, USA).

\section{Results}

\section{Clinical Evaluation Determined the EDS Features}

The family was clinically evaluated by a consultant at $\mathrm{MMCH}$. Affected members of the family manifested typical symptoms of the EDS type 1, including skin loosening (hyperextensivity of the skin), tissue fragility, and bruised skin with variable degree of expression amongst the two members. Unaffected members of the family including normal siblings and parents did not show any of these disease symptoms. A Beighton score of 5 was obtained, indicating a generalized joint hypermobility. Full blood count was normal and clotting disorders were excluded.

\section{Homozygosity Mapping Identified a Loss of Heterozygosity (LOH) Region on Chromosome17q21.33} A common region of homozygosity shared by both the affected members of the family was identified by whole genome SNP genotyping array with the help of 2.5M Illumina iScan platform. Healthy members of the family did not share this homozygosity region. The shard homozygous region is of $4 \mathrm{Mb}$ on chromosome 17q21.33.

\section{WES Analysis Identified a Homozygous Variant in Col1A1 Gene}

An average coverage (covering 214405 exons and splice sites) of $98.3 \%$ was obtained from exome sequencing. Different suitable filter options were employed to analyze WES data generated by Illumina NextSeq500 platform. The shared homozygous region identified by genome-wide SNP genotyping array was primarily targeted due to its disease relevance, in order to identify underlying gene of interest. A missense homozygous variant (c.2050G $>A$ ) in the exon 31 of the COL1A1 gene was identified. The gene COL1A1 is present in the shared homozygous region. Besides targeting this shared homozygous region, different filter options like pathogenicity, allele frequency, genomic position, nonsynonymous variants, protein effect, etc., were used to further filter the entire 90,000 genetic variants. However, we failed to detect any other notable, relevant genetic variants responsible for the EDS phenotype. Therefore, a missense variant (c.2050G > A; p.Glu684Lys) in exon 31 of the COL1A1 gene was considered as the genetic defect underlying EDS phenotype in the family.

\section{Sanger Sequencing Validate the Variant and Segregation Analysis}

The variant present in the exon 31 of the COL1A1 gene was validated by Sanger sequencing approach. Data analysis validated the presence of the variant (c.2050G >A) and its complete segregation within the family. Variant (c.2050G $>$ A) was found in homozygous state in the DNA of affected individuals, while unaffected members of the family were either heterozygous or wild type (-Fig. 1B).

\section{Discussion}

Heterozygous mutations in type 1 collagen gene, COL1A1 and COL1A2, are frequently known to cause osteogenesis imperfecta. ${ }^{34-36}$ Osteogenesis imperfecta type 1 can be confused with EDS syndrome, however, a detailed physical examination along with combination of clinical features can help distinguish the two. ${ }^{37}$

Patients with inherited connective tissue disorders present with diverse clinical features, and differential diagnosis is challenging. ${ }^{23}$ In such cases, clinical distinction is not always straightforward for the physician. We presented a Saudi family with two members afflicted with connective tissue disorders. Complete clinical evaluation diagnosed the individuals with a classic type of Ehlers-Danlos syndrome (cEDS). Patients demonstrated skin fragility as a bruising and poor wound healing. Surgical scars were evident on legs. Interviewing elders revealed presence of individuals with similar clinical features in another loop of the family. Family pedigree was found consistent with an autosomal recessive inheritance pattern.

cEDS with typical disease symptoms included exceptional high skin extensibility and fragility, hypermobility of joints, atrophic scared and bruised skin. Molecular investigations led to the identification of a homozygous missense variant (c.2050G > A) in the COL1A1 gene. This is the second report of a homozygous variant in the COL1A1 in EDS patients. The clinical features including respiratory distress at birth, severe hypotonia with muscle wasting, head lag, and absent reflexes, reported earlier in a family with same homozygous mutation (c.2050G > A) in COL1A1 have not been observed in our cases. This report provides further evidence that COL1A1 homozygous variants may cause EDS like phenotype, and the variant identified in this study is a founder in Saudi population.

\section{Authors' Contributions}

J.A.H, A.H., A.A. performed variant validation using Sanger approach; J.A.H. wrote the initial draft; F.F. recruited family and performed phenotyping; A.A., and S.A. performed DNA extraction and exome libraries preparation; K.R. and S.B. designed the study, analyzed exome data, and wrote the manuscript. All authors read and approved the final manuscript.

\section{Data Availability Statement}

All data including exome sequencing files and Sanger reads are available on request.

Conflict of Interest

None declared.

Acknowledgments

We are thankful to patients and their family members for their participation and contribution in this work.

\section{References}

1 Malfait F, Francomano C, Byers P, et al. The 2017 international classification of the Ehlers-Danlos syndromes. Am J Med Genet C Semin Med Genet 2017;175(01):8-26 
2 Ghali N, Sobey G, Burrows N. Ehlers-Danlos syndromes. BMJ 2019;366:14966

3 Steinmann B, Royce PM, Superti-Furga A. The Ehlers-Danlos syndrome. In: Peter MR, Beat S, eds. Connective Tissue and its Heritable Disorders: Molecular, Genetic, and Medical Aspects. Wiley-Liss, Inc. 2002:431-523

4 Wenstrup RJ, Hoechstetter LB. Ehlers-Danlos syndromes. In: Cassady SB, Allanson JE, eds. Management of Genetic Syndromes. 2;Hoboken: Wiley; 2005:211-223

5 Kapferer-Seebacher I, Pepin M, Werner R, et al;Molecular Basis of Periodontal EDS Consortium. Periodontal Ehlers-Danlos syndrome is caused by mutations in C1R and C1S, which encode subcomponents $\mathrm{C} 1 \mathrm{r}$ and $\mathrm{C} 1 \mathrm{~s}$ of complement. Am J Hum Genet 2016;99(05):1005-1014

6 Tinkle B, Castori M, Berglund B, et al. Hypermobile Ehlers-Danlos syndrome (a.k.a. Ehlers-Danlos syndrome Type III and Ehlers-Danlos syndrome hypermobility type): Clinical description and natural history. Am J Med Genet C Semin Med Genet 2017;175(01):48-69

7 Kosho T, Mizumoto S, Watanabe T, Yoshizawa T, Miyake N, Yamada S. Recent Advances in the Pathophysiology of Musculocontractural Ehlers-Danlos Syndrome. Genes (Basel) 2019;11 (01):43

8 D'Alessio M, Ramirez F, Blumberg BD, et al. Characterization of a COL1A1 splicing defect in a case of Ehlers-Danlos syndrome type VII: further evidence of molecular homogeneity. Am J Hum Genet 1991;49(02):400-406

9 Carr AJ, Chiodo AA, Hilton JM, Chow CW, Hockey A, Cole WG. The clinical features of Ehlers-Danlos syndrome type VIIB resulting from a base substitution at the splice acceptor site of intron 5 of the COL1A2 gene. J Med Genet 1994;31(04):306-311

10 De Paepe A, Nuytinck L, Hausser I, Anton-Lamprecht I, Naeyaert JM. Mutations in the COL5A1 gene are causal in the Ehlers-Danlos syndromes I and II. Am J Hum Genet 1997;60(03):547-554

11 Byers PH, Duvic M, Atkinson M, et al. Ehlers-Danlos syndrome type VIIA and VIIB result from splice-junction mutations or genomic deletions that involve exon 6 in the COL1A1 and COL1A2 genes of type I collagen. Am J Med Genet 1997;72(01):94-105

12 Gilchrist D, Schwarze U, Shields K, MacLaren L, Bridge PJ, Byers PH. Large kindred with Ehlers-Danlos syndrome type IV due to a point mutation (G571S) in the COL3A1 gene of type III procollagen: low risk of pregnancy complications and unexpected longevity in some affected relatives. Am J Med Genet 1999;82(04):305-311

13 Nuytinck L, Freund M, Lagae L, Pierard GE, Hermanns-Le T, De Paepe A. Classical Ehlers-Danlos syndrome caused by a mutation in type I collagen. Am J Hum Genet 2000;66(04):1398-1402

14 Alzahrani F, Alkeraye S, Alkuraya FS. The alternatively spliced exon of COL5A1 is mutated in autosomal recessive classical EhlersDanlos syndrome. Clin Genet 2018;93(04):936-937

15 Dembure PP, Priest JH, Snoddy SC, Elsas LJ. Genotyping and prenatal assessment of collagen lysyl hydroxylase deficiency in a family with Ehlers-Danlos syndrome type VI. Am J Hum Genet 1984;36(04):783-790

16 Burch GH, Gong Y, Liu W, et al. Tenascin-X deficiency is associated with Ehlers-Danlos syndrome. Nat Genet 1997;17(01):104-108

17 Faiyaz-Ul-Haque M, Zaidi SHE, Al-Ali M, et al. A novel missense mutation in the galactosyltransferase-I (B4GALT7) gene in a family exhibiting facioskeletal anomalies and Ehlers-Danlos syndrome resembling the progeroid type. Am J Med Genet A 2004; 128A(01):39-45

18 Colige A, Nuytinck L, Hausser I, et al. Novel types of mutation responsible for the dermatosparactic type of Ehlers-Danlos syndrome (Type VIIC) and common polymorphisms in the ADAMTS2 gene. J Invest Dermatol 2004;123(04):656-663

19 Giunta C, Chambaz C, Pedemonte M, Scapolan S, Steinmann B. The arthrochalasia type of Ehlers-Danlos syndrome (EDS VIIA and
VIIB): the diagnostic value of collagen fibril ultrastructure. Am J Med Genet A 2008;146A(10):1341-1346

20 Janecke AR, Li B, Boehm M, et al. The phenotype of the musculocontractural type of Ehlers-Danlos syndrome due to CHST14 mutations. Am J Med Genet A 2016;170A(01):103-115

21 Baumann M, Giunta C, Krabichler B, et al. Mutations in FKBP14 cause a variant of Ehlers-Danlos syndrome with progressive kyphoscoliosis, myopathy, and hearing loss. Am J Hum Genet 2012;90(02):201-216

22 Aldeeri AA, Alazami AM, Hijazi H, Alzahrani F, Alkuraya FS Excessively redundant umbilical skin as a potential early clinical feature of Morquio syndrome and FKBP14-related Ehlers-Danlos syndrome. Clin Genet 2014;86(05):469-472

23 Alazami AM, Al-Qattan SM, Faqeih E, et al. Expanding the clinical and genetic heterogeneity of hereditary disorders of connective tissue. Hum Genet 2016;135(05):525-540

24 Blackburn PR, Xu Z, Tumelty KE, et al. Bi-allelic alterations in AEBP1 lead to defective collagen assembly and connective tissue structure resulting in a variant of Ehlers-Danlos syndrome. Am J Hum Genet 2018;102(04):696-705

25 Malfait F, De Paepe A. The Ehlers-Danlos syndrome. Adv Exp Med Biol 2014;802:129-143

26 Albarry MA, Hashmi JA, Alreheli AQ et al. Novel homozygous lossof-function mutations in RP1 and RP1L1 genes in retinitis pigmentosa patients. Ophthalmic Genet 2019;40(06):507-513

27 Almatrafi A, Alfadhli F, Khan YN, et al. A Heterozygous mutation in the triple helical region of the Alpha 1 (II) chain of the COL2A1 protein causes non-lethal spondyloepiphyseal dysplasia congenita. Genet Test Mol Biomarkers 2019;23(05):310-315

28 Hashmi JA, Almatrafi A, Latif M, Nasir A, Basit S. An 18 bps inframe deletion mutation in RUNX2 gene is a population polymorphism rather than a pathogenic variant. Eur J Med Genet 2019;62 (02):124-128

29 Basit S, Al-Edressi HM, Sairafi MH, et al. Centromere protein (CENPI) is a candidate gene for $\mathrm{X}$-linked steroid sensitive nephrotic syndrome. J Nephrol 2020;33(04):763-769

30 Kõressaar T, Lepamets M, Kaplinski L, Raime K, Andreson R, Remm M. Primer3_masker: integrating masking of template sequence with primer design software. Bioinformatics 2018;34(11):1937-1938

31 Alharby E, Albalawi AM, Nasir A, et al. A homozygous potentially pathogenic variant in the PAXBP1 gene in a large family with global developmental delay and myopathic hypotonia. Clin Genet 2017;92(06):579-586

32 Hashmi JA, Al-Harbi KM, Ramzan K, et al. A novel splice-site mutation in the ASPM gene underlies autosomal recessive primary microcephaly. Ann Saudi Med 2016;36(06):391-396

33 Basit S, Albalawi AM, Alharby E, Khoshhal KI. Exome sequencing identified rare variants in genes HSPG2 and ATP2B4 in a family segregating developmental dysplasia of the hip. BMC Med Genet 2017;18(01):34

$34 \mathrm{Xi} \mathrm{L}$, Zhang H, Zhang Z-L. Clinical and genetic analysis in 185 Chinese probands of osteogenesis imperfecta.J Bone Miner Metab 2020 (e-pub ahead of print). Doi: 10.1007/s00774-020-01163-5

35 Alkhiary YM, Ramzan A, Ilyas M, et al. Whole exome sequencing analysis identifies a missense variant in COL1A2 gene which causes osteogenesis imperfecta Type IV in a family from Saudi Arabia. J Musculoskelet Surg Res 2017;1(02):33-38

36 Basit S. Next-Generation sequencing and molecular diagnosis in musculoskeletal disorders. J Musculoskelet Surg Res. 2017;1(02): 23-24

37 Colombi M, Dordoni C, Chiarelli N, Ritelli M. Differential diagnosis and diagnostic flow chart of joint hypermobility syndrome/ ehlers-danlos syndrome hypermobility type compared to other heritable connective tissue disorders. Am J Med Genet C Semin Med Genet 2015;169C(01):6-22 